\title{
A NONARCHIMEDEAN THEORY OF ANALYTIC CONTINUATION IN SEVERAL VARIABLES
}

\author{
A. I. THALER
}

Abstract. Recently B. Dwork proved the validity of the functional equation, conjectured by A. Weil, for a nonsingular projective hypersurface defined over a finite field. The proof made use of work of M. Krasner, wherein a uniqueness theorem for an analog of analytic continuation in ultrametric spaces is proved. The methods involved give information concerning the behavior of the undetermined factor \pm 1 in the functional equation for such a hypersurface if one of the coefficients of the polynomial is varied. In this paper, Krasner's result is extended to a uniqueness theorem for analytic elements in $n$ variables. This result will be applied to the Weil zeta function in a later work.

1. Preliminaries. Let $\Omega$ be an algebraically closed field complete with respect to a nonarchimedean rank one valuation $x \rightarrow$ ord $x$ with value group $\mathfrak{G} \subset \boldsymbol{R}$, where $\boldsymbol{R}$ denotes the additive group of real numbers. We shall assume that $\mathfrak{G}$ is dense in $\boldsymbol{R}$. For $b \in \boldsymbol{R}$, we define $\Gamma_{b}=\{\boldsymbol{\xi} \in \boldsymbol{R}$ : ord $\xi=b\}$. Let $\mathfrak{D}$ denote the valuation ring of $\Re, \mathfrak{D}=\bigcup_{b \geqq 0} \Gamma_{b}$, and let $\mathfrak{P}$ denote the ideals of nonunits in $\mathfrak{D}, \mathfrak{P}=\bigcup_{b>0} \Gamma_{b}$. It will occasionally be convenient to use the notation $|x|=p^{\text {ord } x}$, where $p$ is the characteristic of the residue class field of $\Omega$, denoted by $k$.

The following definition is due to Krasner [2].

Definition 1.1. Let $D$ be a subset of the "projective field" $\Omega^{*}=$ $\Omega \cup\left\{\infty^{*}\right\}$. We say that $D$ is a quasi-connected domain of $\Omega^{*}$ if, for every $\alpha \in D \cap \Omega$, the following property is satisfied: for every $\xi \in D$, the set of real numbers

$$
H_{\xi}=\{|x-\alpha|: x \in \Omega-D,|x-\alpha|<|\xi-\alpha|\}
$$

is a finite set.

LEMMA 1.2. Let $\zeta_{1}, \zeta_{2}, \cdots, \zeta_{r}$ be distinct elements of $\mathfrak{D}$; then there is an element $\xi$ of $D$ such that $\left|\xi-\zeta_{i}\right|=1$ for $i=1,2, \cdots, r$.

This is a special case of Lemma 1 of [3], and so we may omit the proof.

Received by the editors September 3, 1970.

AMS 1970 subject classifications. Primary 12B30; Secondary 12J10, 32B99.

Key words and phrases. Weil zeta function, quasi-connected, axially quasi-connected, nonarchimedean analytic continuation.

(c) American Mathematical Society 1972 
Proposition 1.3. Let $f(x) \in \mathfrak{D}[x], f \not \equiv 0$. For any positive number $\delta$, the sets

$$
W_{\delta}(f)=\{\xi \in \mathfrak{D}:|f(\xi)|>\delta\}, \quad W_{\delta}^{\#}(f)=\{\xi \in \mathfrak{D}:|f(\xi)| \geqq \delta\}
$$

are quasi-connected.

Proof. Let $f(x)=\left(x-\zeta_{1}\right)^{e_{1}} \cdots\left(x-\zeta_{r}\right)^{e_{r}}\left(x \beta_{1}-1\right)^{c_{1}} \cdots\left(x \beta_{s}-1\right)^{c_{s}}$, $\zeta_{1}, \zeta_{2}, \cdots, \zeta_{r}$ distinct elements of $\mathfrak{D}, \beta_{1}, \beta_{2}, \cdots, \beta_{s}$ distinct nonunits in $\mathfrak{D}$. For $\delta \geqq 0$, let $\mathcal{R}_{\delta}, \mathcal{R}_{\delta}^{\#}$ be sets of real $r$-tuples defined by

$$
\begin{aligned}
& \mathcal{R}_{\delta}=\left\{\left(\delta_{1}, \cdots, \delta_{r}\right): \delta_{1}^{e_{1}} \cdots \delta_{r}^{e_{r}}>\delta, 0 \leqq \delta_{i} \leqq 1, i=1, \cdots, r\right\}, \\
& \mathcal{R}_{\delta}^{\#}=\left\{\left(\delta_{1}, \cdots, \delta_{r}\right): \delta_{1}^{e_{1}} \cdots \delta_{r}^{e_{r}} \geqq \delta, 0 \leqq \delta_{i} \leqq 1, i=1, \cdots, r\right\},
\end{aligned}
$$

and, for any $r$-tuple $\left(\delta_{1}, \cdots, \delta_{r}\right)$, let $W\left(\delta_{1}, \cdots, \delta_{r}\right)=\left\{\xi \in \mathfrak{D}:\left|\xi-\zeta_{i}\right| \geqq\right.$ $\left.\delta_{i}, i=1,2, \cdots, r\right\}$. Since, as is clear from Definition 1.1, a disk from which finitely many (open or closed) disks have been removed is a quasiconnected domain, it follows that, for any $r$-tuple $\left(\delta_{1}, \cdots, \delta_{r}\right)$, the set $W\left(\delta_{1}, \cdots, \delta_{r}\right)$ is quasi-connected.

Let us consider the collections

$$
\begin{aligned}
\mathcal{C}_{\delta} & =\left\{W\left(\delta_{1}, \cdots, \delta_{r}\right):\left(\delta_{1}, \cdots, \delta_{r}\right) \in \mathcal{R}_{\delta}\right\}, \\
\mathcal{C}_{\delta}^{\#} & =\left\{W\left(\delta_{1}, \cdots, \delta_{r}\right):\left(\delta_{1}, \cdots, \delta_{r}\right) \in \mathcal{R}_{\delta}^{\#}\right\} .
\end{aligned}
$$

It is noted that, for any $\delta, \mathrm{C}_{\delta}$ is a subfamily of $\mathrm{C}_{\delta}^{\#}$, and that $\mathrm{C}_{\delta}$ (respectively $\mathrm{C}_{\delta}^{\#}$ ) is an empty family of sets if $\delta \geqq 1$ (respectively $\delta>1$ ). We now recall that, in the terminology of Krasner, a family $F$ of sets is said to be linked if any two sets $A, B$ of $F$ can be joined by a chain, that is to say a finite collection $A=C_{0}, C_{1}, \cdots, C_{m}=B$ of sets of the family such that any two consecutive terms $C_{i-1}, C_{i}$ are nondisjoint, and we assert that the collections $\mathcal{C}_{\delta}, \mathcal{C}_{\delta}^{\#}$ are either empty or linked families of quasi-connected sets. In fact, we are able to prove a stronger statement, namely that for any choice of $\delta$ in the closed unit interval, there is an element $\xi \in \mathfrak{D}$ common to each member of the family $\mathcal{C}_{\delta}^{\#}$. For, according to Lemma 1.2, an element $\xi$ of $D$ may be chosen satisfying $\left|\xi-\zeta_{i}\right|=1, i=1,2, \cdots, r$, and therefore, since $\left(\delta_{1}, \cdots, \delta_{r}\right) \in \mathrm{C}_{\delta}^{\#}$ entails $\delta_{i} \leqq 1$ for all $i$, the assertion follows. But then, by a theorem of Krasner in the cited reference, the sets $\bigcup_{W \in \mathcal{C}_{\delta}} W, \bigcup_{W \in \mathcal{C}_{\delta}^{\#}} W$ are quasi-connected, for any nonnegative $\delta$ (note that the empty set is trivially a quasi-connected domain). Our desired result then follows from the observations that these latter unions are the sets $W_{\delta}(f)$ and $W_{\delta}^{\#}(f)$, respectively.

Definition 1.4. Let $V$ be a subset of $\Omega^{n}, j$ a positive integer, $1 \leqq$ $j \leqq n$, and $\left(a_{1}, a_{2}, \cdots, a_{n-1}\right) \in \mathfrak{\Omega}^{n-1}$. The symbol $V^{(j)}\left(a_{1}, \cdots, a_{n-1}\right)$ denotes the subset of $\Omega$ defined by

$$
V^{(j)}\left(a_{1}, \cdots, a_{n-1}\right)=\left\{\alpha \in \Re:\left(a_{1}, \cdots, a_{j-1}, \alpha, a_{j}, \cdots, a_{n-1}\right) \in V\right\} .
$$


If $V$ has the property that, for each integer $j, 1 \leqq j \leqq n$, and for each $(n-1)$-tuple $\left(a_{1}, \cdots, a_{n-1}\right) \in \Omega^{n-1}$, the set $V^{(j)}\left(a_{1}, \cdots, a_{n-1}\right)$ is a quasiconnected domain, the set $V$ is said to be axially quasi-connected.

Corollary 1.5. For $R\left(X_{1}, X_{2}, \cdots, X_{n}\right) \in \mathfrak{D}\left[X_{1}, X_{2}, \cdots, X_{n}\right]$ let $W=\left\{\left(\xi_{1}, \xi_{2}, \cdots, \xi_{n}\right) \in \mathfrak{D}^{n}:\right.$ ord $\left.R\left(\xi_{1}, \xi_{2}, \cdots, \xi_{n}\right)=0\right\}$. Then the set $W$ is axially quasi-connected.

Proof. Let $j$ be any integer between 1 and $n$, and let $R_{j}^{*}(x) \in \mathfrak{D}[x]$ be defined by

$$
R_{j}^{*}(x)=R\left(a_{1}, \cdots, a_{j-1}, x, a_{j}, \cdots, a_{n-1}\right),
$$

where $a_{1}, \cdots, a_{n-1}$ are arbitrarily chosen elements of $\mathfrak{D}$; then $W^{(j)}\left(a_{1}, \cdots, a_{n-1}\right)$ is either empty or equal to $W_{1}^{\#}\left(R_{j}^{*}\right)$, and the preceding proposition applies.

2. Uniqueness theorem. In this section, a uniqueness theorem for analytic elements in several variables, generalizing the one-variable theory of Krasner, is proved. We do not claim to have a completely satisfactory generalization of Krasner's concept of a quasi-connected domain; in particular, while it is not sufficient only to assume that a subset of $\boldsymbol{\Omega}^{n}$ be axially quasi-connected, it seems as though our definition of $W$ in the statement of the theorem is overly restrictive. However, it is only regions so defined with which we will be concerned in [4].

It is necessary to introduce some new ideas before the uniqueness theorem is stated.

Definition 2.1. Let $\xi=\left(\xi_{1}, \xi_{2}, \cdots, \xi_{n}\right), \eta=\left(\eta_{1}, \eta_{2}, \cdots, \eta_{n}\right)$ be a pair of elements of $\Omega^{n}$; we say that $\xi$ is directly axially joined to $\eta$ if $\xi_{i}=\eta_{i}$ for all but possibly one of the indices $i=1,2, \cdots, n$. If $U$ is a subset of $\Omega^{n}$, and if $\xi, \eta$ are elements of $U$, we say that $\xi$ and $\eta$ are $U$ axially joined if there is a sequence $\eta=\xi^{(0)}, \xi^{(1)}, \cdots, \xi^{(N)}=\xi$ with the property that, for $i=0,1,2, \cdots, N, \xi^{(i)} \in U$, and, for $i=1,2, \cdots, N$, $\xi^{(i-1)}$ is directly axially joined to $\xi^{(i)}$.

It is clear from the definition that "is $U$-axially joined to" is an equivalence relation.

Definition 2.2. For $U \subset W \subset \Re^{n}$, we define the axial join of $U$ in $W$, $W^{\prime}$, by

$$
W^{\prime}=\{\xi \in W: \xi \text { is } W \text {-axially joined to an element of } U\} .
$$

Proposition 2.3. If $R\left(X_{1}, X_{2}, \cdots, X_{n}\right) \in \mathfrak{D}\left[X_{1}, X_{2}, \cdots, X_{n}\right]$, ord $R(0,0, \cdots, 0)=0$, let $W=\left\{\left(\xi_{1}, \cdots, \xi_{n}\right) \in \mathfrak{D}^{n}\right.$ :ord $R\left(\xi_{1}, \cdots, \xi_{n}\right)=$ $0\}$ and let $\rho_{1}, \rho_{2}, \cdots, \rho_{n}$ be a set of positive numbers such that $U=$ $\Gamma_{\rho_{1}} \times \Gamma_{\rho_{2}} \times \cdots \times \Gamma_{\rho_{n}}$ is not empty. Then, if $W^{\prime}$ denotes the axial join of $U$ in $W, W^{\prime}=W$. 
Proof. If $n=1$, any two elements of $\Re$ are directly axially joined, and so $W^{\prime}=W$ trivially.

Assume the validity of the proposition for polynomials in $n-1$ variables with coefficients in $\mathfrak{D}, n \geqq 2$, and let $\left(\psi_{1}, \cdots, \psi_{n}\right) \in W$. We shall construct an element $\left(\eta_{1}, \eta_{2}, \cdots, \eta_{n}\right) \in U$ which is $W$-axially joined to $\left(\psi_{1}, \psi_{2}, \cdots, \psi_{n}\right)$.

Consider the image $\bar{R}\left(X_{1}, \cdots, X_{n}\right)$ of $R\left(X_{1}, \cdots, X_{n}\right)$ under the residue class map: it follows from the definition of $W$ that, if $\bar{\xi}_{i}$ denotes the residue class of $\xi_{i}$ under reduction $\bmod \mathfrak{P},\left(\xi_{1}, \cdots, \xi_{n}\right)$ is an element of $W$ if and only if $\bar{R}\left(\bar{\xi}_{1}, \cdots, \bar{\xi}_{n}\right) \neq 0$. Let the polynomials $\bar{R}^{\prime}, \bar{R}_{0}^{\prime}$ in $k\left[X_{n}\right]$ be defined by

$$
\bar{R}_{0}^{\prime}\left(X_{n}\right)=\bar{R}\left(0,0, \cdots, 0, X_{n}\right), \quad \bar{R}^{\prime}\left(X_{n}\right)=\bar{R}\left(\bar{\psi}_{1}, \bar{\psi}_{2}, \cdots, \bar{\psi}_{n-1}, X_{n}\right) ;
$$

since $\bar{R}_{0}^{\prime}(0) \bar{R}^{\prime}\left(\bar{\psi}_{n}\right) \neq 0$, the product of these two polynomials is not the zero polynomial. But $k$ is infinite, so the existence of an element $\eta$ of $\mathfrak{D}$ with the property $\bar{R}^{\prime}(\bar{\eta}) \bar{R}_{0}^{\prime}(\bar{\eta}) \neq 0$ is guaranteed.

Let $R^{*}\left(X_{1}, X_{2}, \cdots, X_{n-1}\right)=R\left(X_{1}, X_{2}, \cdots, X_{n-1}, \eta\right)$ and put $W^{*}=$ $\left\{\left(\xi_{1}, \xi_{2}, \cdots, \xi_{n-1}\right) \in D^{n-1}\right.$ :ord $\left.R^{*}\left(\xi_{1}, \xi_{2}, \cdots, \xi_{n-1}\right)=0\right\}$; then ord $R^{*}(0,0, \cdots, 0)=0$ and $\left(\psi_{1}, \psi_{2}, \cdots, \psi_{n-1}\right) \in W^{*}$, and therefore, by the induction hypothesis, $\left(\psi_{1}, \psi_{2}, \cdots, \psi_{n-1}\right)$ is $W^{*}$-axially joined to an element $\left(\eta_{1}, \eta_{2}, \cdots, \eta_{n-1}\right) \in \Gamma_{\rho_{1}} \times \Gamma_{\rho_{2}} \times \cdots \times \Gamma_{\rho_{n-1}}$. Thus, if we choose any element $\eta_{n}$ of $\Gamma_{\rho_{n}}$, the conclusion follows from the fact that $\left(\psi_{1}, \psi_{2}, \cdots, \psi_{n-1}, \eta\right)$ and $\left(\psi_{1}, \psi_{2}, \cdots, \psi_{n}\right)$ are directly axially joined, $\left(\eta_{1}, \eta_{2}, \cdots, \eta_{n-1}, \eta\right)$ and $\left(\eta_{1}, \eta_{2}, \cdots, \eta_{n}\right)$ are axially joined, and $\left(\xi_{1}, \xi_{2}, \cdots, \xi_{n-1}\right) \in W^{*}$ if and only if $\left(\xi_{1}, \xi_{2}, \cdots, \xi_{n-1}, \eta\right) \in W$.

REMARK 2.4. If $\xi=\left(\xi_{1}, \xi_{2}, \cdots, \xi_{n}\right), \quad \eta=\left(\eta_{1}, \eta_{2}, \cdots, \eta_{n}\right)$ are elements of $\mathfrak{D}^{n}$, and if $\bar{\xi}_{i}=\bar{\eta}_{i}, i=1,2, \cdots, n$, then $\xi \in W$ if and only if $\eta \in W$.

TheOREM 2.5. For $R\left(X_{1}, X_{2}, \cdots, X_{n}\right) \in \mathcal{D}\left[X_{1}, X_{2}, \cdots, X_{n}\right]$, $R(0,0, \cdots, 0) \in \Gamma_{0}$, let $W=\left\{\left(\xi_{1}, \cdots, \xi_{n}\right) \in \mathfrak{D}^{n}:\right.$ ord $\left.R\left(\xi_{1}, \cdots, \xi_{n}\right)=0\right\}$, and let $\left\{f_{m}\left(X_{1}, X_{2}, \cdots, X_{n}\right)\right\},\left\{g_{m}\left(X_{1}, X_{2}, \cdots, X_{n}\right)\right\}, m=1,2,3, \cdots$, be sequences of rational functions defined on $W$ and converging uniformly to functions $f\left(X_{1}, X_{2}, \cdots, X_{n}\right)$ and $g\left(X_{1}, X_{2}, \cdots, X_{n}\right)$, respectively, on $W$. Suppose, for some set of positive numbers $\rho_{1}, \rho_{2}, \cdots, \rho_{n}$, the set $U=$ $\Gamma_{\rho_{1}} \times \Gamma_{\rho_{2}} \times \cdots \times \Gamma_{\rho_{n}}$ is not empty and $f\left(X_{1}, X_{2}, \cdots, X_{n}\right)=$ $g\left(X_{1}, X_{2}, \cdots, X_{n}\right)$ on $U$. Then $f\left(X_{1}, X_{2}, \cdots, X_{n}\right)=g\left(X_{1}, X_{2}, \cdots, X_{n}\right)$ identically on $W$.

Proof. Let $\xi$ be any element of $W$. Then, by Proposition 2.3, there is an element $\eta$ of $U$ and a sequence $\eta=\xi^{(0)}, \xi^{(1)}, \cdots, \xi^{(N)}=\xi$ of elements of $W$ such that adjacent members of the sequence are directly axially joined. The theorem will follow from construction of a sequence 
$\Xi^{(0)}, \Xi^{(1)}, \cdots, \Xi^{(N)}$ of sets, each of which satisfies the conditions:

(1) $\Xi^{(i)} \subset W$.

(2) For any choice of $j, 1 \leqq j \leqq n$, and any element $\xi=\left(\xi_{1}, \xi_{2}, \cdots, \xi_{n}\right)$ of $\Xi^{(i)}$, the set

$$
\Xi_{\xi, j}^{(i)}=\left\{\hat{\xi}_{j}:\left(\xi_{1}, \xi_{2}, \cdots, \xi_{j-1}, \hat{\xi}_{j}, \xi_{j+1}, \cdots, \xi_{n}\right) \in \Xi^{(i)}\right\}
$$

has $\xi_{j}$ as a limit point.

(3) $\xi^{(i)} \in \Xi^{(i)}$.

(4) If $\left(\xi_{1}, \cdots, \xi_{n}\right) \in \Xi^{(i)}$, then $f\left(\xi_{1}, \cdots, \xi_{n}\right)=g\left(\xi_{1}, \cdots, \xi_{n}\right)$.

Such a sequence is constructed inductively. If the initial member $\Xi^{(0)}$ is set equal to $U$, it follows from the hypothesis that $\Xi^{(0)}$ satisfies the four conditions.

Now, let $0 \leqq i<N$, and suppose the set $\Xi^{(i)}$ has been chosen in such a manner that conditions (1)-(4) are satisfied. Let

$$
\begin{aligned}
\Xi^{(i+1)}=\bigcup_{j=1}^{n}\left\{\left(\xi_{1}, \xi_{2}, \cdots, \xi_{j-1}, \eta_{j}, \xi_{j+1}, \cdots, \xi_{n}\right) \in W:\right. & \text { for some } \left.\xi_{j},\left(\xi_{1}, \cdots, \xi_{j}, \cdots, \xi_{n}\right) \in \Xi^{(i)}\right\} .
\end{aligned}
$$

It is obvious that $\Xi^{(i+1)}$ so defined satisfies the first of our conditions.

Suppose $\psi=\left(\psi_{1}, \cdots, \psi_{n}\right) \in \Xi^{(i+1)}$; if $j$ is any integer, $1 \leqq j \leqq n$, we must show that $\Xi_{\psi, j}^{(i+1)}$ has $\psi_{j}$ as a limit point. But $\psi \in \Xi^{(i+1)}$ implies the existence of an integer $j^{\prime}, 1 \leqq j^{\prime} \leqq n$, and an element $\xi=\left(\xi_{1}, \cdots, \xi_{n}\right) \in$ $\Xi^{(i)}$ such that $\psi_{i}=\xi_{i}$ if $i \neq j^{\prime}$. If $j^{\prime}=j$, Remark 2.4 implies that all elements congruent to $\psi_{j} \bmod \mathfrak{B}$ are in $\Xi_{\psi, j}^{(i+1)}$, and so $\psi_{j}$ is certainly a limit point of this latter set. On the other hand, if $j^{\prime} \neq j$, we use the fact that $\xi_{j}$ is a limit point of $\Xi_{\xi, j}^{(i)}$. Thus, we can choose an infinite subset $\left\{\hat{\xi}_{j, l}\right\}, l=0,1,2, \cdots$, of $\Xi_{\xi, j}^{(i)}$ such that $\hat{\xi}_{j, l} \rightarrow \xi_{j}$ as $l \rightarrow \infty$, and such that these elements are all in the same residue class $\bmod \mathfrak{B}$; but then, if we define $\phi_{l}=\left(\phi_{l 1}, \phi_{l 2}, \cdots, \phi_{l n}\right)$ by

$$
\begin{aligned}
\phi_{l i} & =\psi_{j^{\prime}} & & \text { if } i=j^{\prime}, \\
& =\hat{\xi}_{j, l} & & \text { if } i=j, \\
& =\xi_{i} & & \text { otherwise, }
\end{aligned}
$$

Remark 2.4 implies that $\phi_{l} \in \Xi^{(i+1)}$ for all $l$, and, as $l \rightarrow \infty, \phi_{l j} \rightarrow \xi_{j}=\psi_{j}$, from which it follows that condition (2) is satisfied by the set $\Xi^{(i+1)}$.

Condition (3) is fulfilled since $\xi^{(i)} \in \Xi^{(i)}$ and $\xi^{(i)}, \xi^{(i+1)}$ are directly axially joined.

Finally, if $\psi=\left(\psi_{1}, \cdots, \psi_{n}\right) \in \Xi^{(i+1)}$, we choose $\xi=\left(\xi_{1}, \cdots, \xi_{n}\right)$ and the integer $j$ such that $\psi_{i}=\xi_{i}$ if $i \neq j$. Let

$$
R_{\psi, j}(X)=R\left(\psi_{1}, \psi_{2}, \cdots, \psi_{j-1}, X, \psi_{j+1}, \cdots, \psi_{n}\right),
$$

and let $W_{\psi, j}=\left\{\eta_{j}\right.$ :ord $\left.R_{\psi, j}\left(\eta_{j}\right)=0\right\}$. Proposition 2.3 tells us that $W_{\psi, j}$ is a quasi-connected domain; but, by the induction hypothesis, 
$f\left(\xi_{1}, \cdots, \xi_{j-1}, X, \xi_{j+1}, \cdots, \xi_{n}\right)$ is identically equal to

$$
g\left(\xi_{1}, \cdots, \xi_{j-1}, X, \xi_{j+1}, \cdots, \xi_{n}\right)
$$

on the set $\Xi_{\xi, j}^{(i)}$; since this subset of $W_{\psi, j}$ has a limit point in itself, application of the one-variable uniqueness theorem proved by Krasner gives the result that

$$
f\left(\xi_{1}, \cdots, \xi_{j-1}, X, \xi_{j+1}, \cdots, \xi_{n}\right)=g\left(\xi_{1}, \cdots, \xi_{j-1}, X, \xi_{j+1}, \cdots, \xi_{n}\right)
$$

identically on $W_{\psi, j}$. This proves our theorem.

\section{REFERENCES}

1. B. M. Dwork, On the zeta function of a hypersurface. II, Ann. of Math. (2) 80 (1964), 227-299. MR 32 \#5654.

2. M. Krasner, Prolongement analytique dans les corps valués complets: domaines quasi connexes, C.R. Acad. Sci. Paris 238 (1954), 2385-2387. MR 16, 799.

, Prolongement analytique dans les corps valués complets: éléments analytiques, préliminaires du théorème d'unicité, C.R. Acad. Sci. Paris 239 (1954), 468-470. MR 16, 799.

- Prolongement analytique dans les corps valués complets: démonstration de la loi d'unicité: fonctions analytiques uniformes, C.R. Acad. Sci. Paris 239 (1954), 745-747. MR 16, 799.

- Prolongement analytique dans les corps valués complets: préservation de l'analyticité par les opérations rationnelles, C.R. Acad. Sci. Paris 244 (1957), 1304-1306. MR 19, 395; 28 p. 1246.

- Prolongement analytique dans les corps valués complets: préservation de l'analyticité par les opérations rationnelles: quasi-connexité et éléments analytiques réguliers, C.R. Acad. Sci. Paris 244 (1957), 1599-1602. MR 19, 395; 28 p. 1246.

- Prolongement analytique dans les corps valués complets: uniformité des fonctions analytiques: l'analyticité des fonctions méromorphes, C.R. Acad. Sci. Paris 244 (1957), 1996-1999. MR 19, 395.

- Prolongement analytique dans les corps valués complets: préservation de l'analyticité par la convergence uniforme et par la dérivation: théorème de Mittag-Löffler généralisé pour les éléments analytiques, C.R. Acad. Sci. Paris 244 (1957), 2570-2573. MR 19, 395.

- Prolongement analytique dans les corps valués complets: démonstration du théorème de Mittag-Löffler: singularités au bord, C.R. Acad. Sci. Paris 245 (1957), 1285-1288. MR 19, 395.

3. A. I. Thaler, On the Newton polytope, Proc. Amer. Math. Soc. 15 (1964), 944-950. MR 29 \#4759.

4. - A multiple-variable deformation theory for a non-singular hypersurface (to appear).

5. M. Krasner, Prolongement analytique uniforme et multiforme dans les corps valués complets, Les Tendances Géométriques en Algèbre et Théorie des Nombres, Éditions du Centre National de la Recherche Scientifique, Paris, 1966, pp. 97-141. MR 34 \#4246.

Department of Mathematics, University of Maryland, College Park, MaryLAND 20742

Current address: Mathematical Sciences Section, National Science Foundation, Washington, D.C. 20550 